\title{
Research on Horizontal Patchwork Connection Technology of Fabricated Shear Wall
}

\author{
Junqiang Han \\ The Engineering \& Technical College of Chengdu University of Technology, Leshan, Sichuan, \\ 614000
}

Keywords: Fabricated Shear Wall, Horizontal Patchwork Connection, Force Analysis

\begin{abstract}
The structure of shear wall has become one of the most commonly used structures for the present residence in our country. The wide application of the structure of the shear wall structure will play an important role in the deepening development of China's housing industry. For the structure of the shear wall structure, the reliability of the connection between the shear wall panels is the key to the seismic performance of the whole structural system, and it is also an important factor in the application of the assembly shear wall structure. In this paper, through the systematic analysis and comparison of the traditional connection technology, a new connection method is proposed, that is, the longitudinal reinforcement is made into a closed U-shaped bar to complete the connection, which is designed to improve the traditional connection structure, Which is conducive to the promotion of defects, and carried out the seismic performance of the experimental study. A total of two prefabricated shear wall horizontal connection nodes and two quasi-static tests with two joint joints with overlapped floors were used to test the yield and damage of the specimen, the stress transmission of the connected bars and the stress distribution of the complex nodes. The characteristics of the system were analyzed and studied. The test results show that the failure mode of the specimen is the bending shear of the shear wall, and the damage process includes the longitudinal steel yield, fracture, crack expansion and concrete peeling. The stress transfer effect of the longitudinal connection of the shear wall is good, and the whole specimen shows a good energy dissipation effect.
\end{abstract}

\section{Introduction}

With the shortage of resources and energy caused by excessive consumption, the traditional construction site and construction technology to bring high-intensity manual labor and high-risk mode of operation to the site construction workers with great physical damage and the "labor shortage" phenomenon, It shows that the high energy consumption and labor-intensive production methods in the construction industry have been incompatible with the theme of low-carbon and environmental protection. At this point, the concept of "housing industrialization" came into being to solve the problem of rapid and energy- Quality of the house. "Housing industry" concept was first proposed by the Japanese experts in 1968, the development has been formed to the structural system of industrialization, structural parts of the general-oriented, through the residential structure of standardized design combined with modern scientific organization and management of the form, the residential Industrial system integration into a development, design, production, assembly, management and other aspects of the perfect system, which will become the cause of the industrialization of housing production, supply, sales and service integration of the production organization.

\section{Advantages and Disadvantages of Prefabricated Concrete Structures}

Prefabricated concrete structure has the advantages of high construction efficiency, good quality, energy saving and environmental protection, and can shorten the construction period, reduce the artificial output, meet the industrialization process of modularization, standardization requirements, is recognized low carbon development technology, prefabricated concrete The advantages of the structure are mainly reflected in: 
Prefabricated concrete components through the factory professional production line, not only the production conditions are superior, and the conservation environment controllable, so the quality and accuracy of component products easy to meet the needs. After the data show that the prefabricated concrete production plant production of concrete products in the strength, compactness, durability, water resistance and other aspects of the concrete than the pouring of concrete products are more assured.

Prefabricated concrete products in the factory using mechanized, automated production equipment production, its production efficiency than the cast-in-place concrete production methods have greatly improved. In addition, the construction work of the prefabricated components in the field is also the widespread use of mechanized equipment to complete the lifting and installation work, by reducing the construction site of the wet work to reduce the amount of scaffolding support and concrete conservation of the time occupied by the weather and less affected , The use of prefabricated concrete technology can significantly shorten the construction period. The production of components in a prefabricated plant can effectively reduce the amount of water and formwork; field installation operations can also effectively reduce noise and emissions of harmful gases. Artificial savings and is conducive to the re-allocation of social resources.

As the prefabricated components are assembled in the field, the installation of the site is difficult to ensure the quality of the connection, which makes the connection between the prefabricated components easily form a weak cross-section, in the past few times in the world of high-intensity earthquake, prefabricated concrete structure Of the overall collapse of the phenomenon is more serious, because the load under the joint node failure is often broken before the destruction of prefabricated components, it is more likely to lead to the collapse of the entire structural system, or even the overall collapse. The challenge of the precast structure of the prefabricated structure has seriously restricted the application of precast concrete structure in the earthquake area. As the prefabricated technology is still in the research stage, the structure of the existing prefabricated connection nodes is relatively complex and the design is more difficult. The structural design staff put forward higher requirements. In addition, the low fault tolerance of prefabricated concrete structure design also makes most designers discouraged, so the immature design level is also an important reason for restricting the application of precast concrete structure.

The widespread use of prefabricated concrete requires pre-construction of prefabricated components factory, which requires early on the initial site, production equipment and other aspects of huge investment. And the current prefabricated structure of the assembly rate is low, limited scope of application and other factors are not enough to bring huge economic benefits.

\section{Nonlinear Finite Element Analysis of Horizontal Connection Joints of Fitting Shear Wall}

The separation model is to define the reinforcement and concrete as different units. Steel bars generally use linear rod units. The concrete unit can select the surface element or body unit according to the calculated dimension. Because of the difference of the two kinds of element stiffness matrices, the deformation of the steel element and the concrete element is not consistent under the action of the load. Therefore, in the separation model, there are usually connection units between the two units of concrete and steel There are rigid connection and connection unit connection in two ways, rigid connection method is to assume that the connection between the concrete and the reinforcement between the anchor is very good, under the action of the load will not appear relative slip, and that the cracks through the concrete unit itself form. And between concrete units and between concrete and steel units are only at the common node at each other. If you want to consider the relative slip between concrete and steel, you need to define the connection unit in the steel unit node and the corresponding location of the concrete unit nodes to set up the connection unit to complete the link.

The discrete crack model deals with the cracking phenomenon by arranging the cracked position on the boundary of the element. When the crack load is reached, the program automatically increases the node at the crack surface or adjusts the original node position, and subdivides it through the cell grid to complete the crack surface and the cell Separation of boundaries. The model 
can simulate the occurrence and development of cracks and the description of the crack width, but the computational complexity of the fractures is limited due to the large amount of fractures. With the development of computer technology, in the future, it is widely used in the plane problem, which is different from the discrete crack model. The distribution fracture model is that the concrete material is treated as anisotropic material. After the concrete is cracked, The crack is simulated by adjusting the constitutive model of concrete. When the integral point of a unit reaches the cracking stress, the stress and strain matrix of the unit material is adjusted to be the whole region. Therefore, the distribution of fracture model is actually the concrete cracks "diffuse into the whole unit", the calculation process to avoid the re-division of the cell grid, reducing the calculation of the workload. It has been very widely used. The crack treatment in ANSYS belongs to this type of fracture.

The method of solving nonlinear equations can be roughly classified into iterative method, incremental method and incremental iterative method. The incremental method divides the load into many increments, and each time a load increment is applied to give the stiffness matrix the different values correspond to the unit stress-strain relationship. The stiffness matrix corresponding to different load increments can have different values, so in a load increment, the stiffness matrix is usually assumed to be constant; the iterative rule is that all loads are applied during each iteration but can not be avoided Error accumulation, so in the subsequent calculation process to gradually change the displacement and strain, so that the analysis unit to meet the nonlinear stress-strain relationship; mixed method using incremental and iterative method, the two methods of organic combination, the load points For multiple incremental load steps, the solution after each step of the load increases is reached by the iterative calculation to achieve equilibrium convergence. This method can better solve the problem that nonlinear calculation results are difficult to converge. The finite element analysis is solved by the mixing method.

ANSYS is used to analyze the non - linear analysis of the joints of the horizontally connected joints and the joint joints of the reinforced concrete assembly shear wall. A separate model is used for the joint specimen of the shear wall, that is, the bar is made of the rod unit LINK8 unit, and the concrete adopts the solid element SOLID65 unit. (BISO) is used to simulate the constitutive relation of steel bar. The multi-linear follow-up hardening model (MKIN) is adopted for the constitutive relation of concrete. The willam-warnke five-parameter criterion is adopted for the failure criterion. The mapping grid is divided into the specimen, and the hybrid method of the incremental method and the iterative method is solved.

In the specimen joint of the shear wall, the local variable cross section of the floor wall has changed the distribution of the stress of the original shear wall, which delayed the cracking and development of the shear wall of the upper part of the floor and made the shear wall The development of cracks in the body after cracking is focused on the upper and lower areas of the floor connection, so the development of the cracks becomes uneven and the rigidity of the whole specimen is obviously improved. From the final failure mode of the specimen, the shear wall The crack width of the wall of the specimen is smaller than that of the horizontal joint, and the cracking of the edge of the shear wall due to the bending deformation and the peeling of the concrete are more intense, which reflects the damage caused by the bending deformation More obvious, the good compression performance of concrete in the joint connection node specimen has been a good play. This phenomenon feedback in the entire assembly of shear wall structure system, the role of the floor will be more obvious in the engineering practice, especially the design stage, put forward the following suggestions:

The failure mechanism of the whole structural system is that the shear wall of the shear wall has the horizontal cracks at the bottom of the shear wall and develops sharply at the bottom of the shear wall, and finally the concrete of the edge of the shear wall is crushed and exited. Therefore, Increasing the configuration of the longitudinal and stirrups of the edge members of the shear wall has a significant effect on improving the bearing capacity of the whole structural system and slowing the cracking of the shear wall.

In the shear wall structure system, the floor itself has a large rigidity in the plane, can play the role of embedded and horizontal force transmission, in the assembly structure, in order to maintain the 
floor of the entire structural system, should avoid the floor and prefabricated shear The wall of the wall caused by sliding and horizontal force transmission failure caused by the adverse situation, it is recommended in the structure of the shear wall structure of the floor to properly reserve a certain amount of wet work, should not use the whole prefabricated concrete floor; prefabricated floor construction joints Should not be vertical joints; basement roof or structure of the roof and other embedded roof on the floor of the embedded and horizontal force transmission depends on the larger parts, should be used in cast-in-place floor, should not use the whole prefabricated or part of the prefabricated floor.

For the floor of a large hole or floor of the local discontinuous situation, need to pay attention to the board with the wall and the wall stiffness of the coordination of the problem. In the case of the wall, the shear wall panels can be increased by increasing the cross-sectional dimension or reinforcement, increasing the piling pillars, the dark columns, etc., because of the small overall stiffness and less likely to crack in the earthquake.

\section{Conclusion}

The structure of the shear wall structure is suitable for the development of China's national conditions and the needs of the development of the housing industry. The wide application of the structural system has milestone significance for the future development of China's construction industry. In this paper, the reliability of the connecting joints of the assembled shear wall structure under the action of the earthquake is systematically studied and some suggestions are put forward for the future design and application of the structure. However, the large-scale application of the assembly shear wall structure system, Still need a lot of theoretical analysis and research work.

\section{References}

[1] Chen Yungang, Liu Jiabin. Study on seismic performance of bolted joint of horizontal shear seam of assembled shear wall [J]. Journal of Harbin Institute of Technology, 2013 (06)

[2] Liu Jiabin, Chen Yungang. Experimental study on seismic behavior of U-shaped closure bar with assembled concrete shear wall [J]. Journal of Southeast University, 2013

[3] Chen Zikang, Zhou Yun. Study and application of assembled concrete frame structure [J]. Engineering earthquake and reinforcement transformation, 2012 (04)

[4] Chen Yaogang, Guo Zhengxing. Technology of joint construction of integral prefabricated shear wall structure [J].Construction Technology, 2011 (11)

[5] Guo Zhengxing, Dong Niancai. Experimental analysis of seismic performance of full prefabricated integral shear wall structure wall [J]. Construction Technology, 2011 (11) 